\title{
FORMAÇÃO DO ACADÊMICO DE ENFERMAGEM: VIVÊNCIA NA ATENÇÃO A USUÁRIOS DE DROGAS PSICOATIVAS
}

\author{
Nursing student's education: experience in the care to users of psychoactive drugs \\ La formación del estudiante de enfermería: vivencia en la atención de los usuarios de \\ drogas
}

Andiara Araújo Cunegundes de Brito ${ }^{1}$

Danielle Souza Silva²

Dulcian Medeiros de Azevedo ${ }^{3}$

\section{RESUMO}

0 artigo objetivou relatar a experiência vivenciada por acadêmicos de enfermagem na Fundação Belo Amor, localizada no município de Caicó/RN, serviço de reabilitação psicossocial para usuários de álcool e outras drogas, buscando reconhecer a rede de saúde mental no município. A vivência partiu da disciplina Políticas Públicas de Saúde Mental, ofertada no sexto período do curso de graduação em enfermagem, e compreendeu dois momentos: a captação da realidade e o desenvolvimento de duas técnicas de grupo. Através das atividades realizadas foi possível aproximar-se da história de vida dos usuários; apreender sentimentos e/ou situações que os levaram ao consumo de drogas ou recaídas; e a vivência do processo de reabilitação atual e as perspectivas futuras. Esta experiência possibilitou que os acadêmicos compreendessem a importância deste serviço para a rede de saúde mental, e ainda para a formação generalista, crítica e reflexiva do profissional enfermeiro.

Palavras-chave: Drogas ilícitas. Serviços de saúde mental. Centros de reabilitação. Educação em enfermagem. Enfermagem psiquiátrica.

\begin{abstract}
This article intended to report the experience lived by nursery nursing academics in Fundação Belo Amor, located in Caicó/RN, a psycho-social rehabilitation service for alcohol and drug users, aiming to recognize the town's mental health network. This living was prompted by the subject of Mental Health Public Policies, offered during the sixth semester of the nursing graduation course, and included two moments: getting to the reality and performing two group techniques. Through the activities performed, it was possible to approach to the users' life-story, to understand their feelings which led them to drugs consumption or recurrences, current rehabilitation process living and future perspectives. This experience promoted the academics to understand the importance of this service to mental health network, and also for generalist, critic, and reflexive training of nursing professionals.
\end{abstract}

Keywords: Illicit Drugs. Mental Health Services. Rehabilitation Centers. Nursing Education. Psychiatric Nursing.

\section{Resumen}

El artículo tiene por objetivo presentar la experiencia de estudiantes de enfermería en la Fundação Belo Amor, ubicado en el municipio de Caicó/RN, servicio de rehabilitación psicosocial para los usuarios de alcohol y otras drogas, con el fin de reconocer la red de salud mental en el municipio. La experiencia viene de la disciplina de Política Pública de Salud Mental, presentada en el sexto semestre del curso de graduación en enfermería, y constaba de dos fases: la captura de la realidad y el desarrollo de dos técnicas de grupo. A través de las actividades realizadas fue posible acercarse a la historia de vida de los usuarios; los sentimientos y/o situaciones que los condujeran al uso de drogas o las recaídas; la experiencia del proceso de rehabilitación actual y de las perspectivas futuras. Esta experiencia posibilitó que los estudiantes entendieran la importancia de este servicio para la red de salud mental, y para la educación en general, crítica y reflexiva del enfermero profesional.

Palabras clave: Drogas llícitas. Servicios de Salud Mental. Centros de Rehabilitación. Educación en Enfermería. Enfermería Psiquiátrica.

\footnotetext{
'Acadêmica do Curso de Graduação em Enfermagem (Licenciatura e Bacharelado), Universidade do Estado do Rio Grande do Norte (UERN), Campus Caicó, Caicó-RN. Brasil. E-mail: andiara_acm@hotmail.com ; ²Acadêmica do Curso de Graduação em Enfermagem (Licenciatura e Bacharelado), UERN, Campus Caicó, Caicó-RN. Brasil. E-mail: daniellerafson@hotmail.com; ${ }^{3}$ Enfermeiro. Mestre em Enfermagem (PGENF-UFRN), Doutorando em Ciências da Saúde (PPGCSa-UFRN). Professor Assistente II, Curso de Graduação em Enfermagem (UERN), Campus Caicó. Líder do Grupo de Pesquisa "A enfermagem no processo saúde-doença individual/coletiva, na educação em saúde e na assistência/gerência de serviços de saúde". Caicó-RN. Brasil. E-mail: professordulcian@gmail.com
} 


\section{INTRODUÇÃO}

A Reforma Psiquiátrica no Brasil surgiu em meados de 1970 com o objetivo de reformular o modelo de atenção em saúde mental, especialmente através da extinção progressiva dos hospitais psiquiátricos e da implantação dos serviços substitutivos, permitindo aos portadores de sofrimento mental uma assistência terapêutica adequada, reabilitação psicossocial e liberdade para usufruir de seus direitos civis. Entre os serviços substitutivos, estabeleceram-se os Centros de Atenção Psicossocial (CAPS), os Ambulatórios de Saúde Mental, os Serviços Residenciais Terapêuticos (SRT), Serviços de Emergência e Urgência, além dos Leitos Psiquiátricos em Hospital Geral.

Os CAPS são serviços abertos e comunitários ligados à assistência diária aos portadores de sofrimento mental, que realizam acompanhamento clínico e buscam a reinserção psicossocial através do acesso ao trabalho, lazer e exercício dos direitos civis. Diferenciando-se pelo porte, capacidade de atendimento e clientela atendida, os CAPS se dividem em cinco modalidades: CAPS I, CAPS II, CAPS III, CAPSi e CAPSad. Desde o ano de 2002, estes serviços experimentam grande expansão através do financiamento e consolidação da Portaria n ${ }^{0} 336$, do Ministério da Saúde.

No município de Caicó, a luta antimanicomial ganhou destaque com a repercussão de casos de maus tratos e violência, denunciados por familiares e pacientes do antigo Hospital Psiquiátrico Milton Marinho, foco de uma intervenção do Ministério da Saúde em 2005. Em julho de 2009, o espaço físico do antigo hospital passou a funcionar como Centro de Atenção Psicossocial (CAPS III "Arte de Viver" - único do Estado), e os moradores do antigo hospital foram alocados em um Serviço Residencial Terapêutico (SRT), atualmente sem credenciamento no Ministério da Saúde.

Além disso, ocorre a tentativa de integração das equipes da Estratégia Saúde da Família (ESF), com enfoque da saúde mental na atenção básica e matriciamento pelo Núcleo de Apoio à Estratégia Saúde da Família (NASF), a espera de instalação de uma CAPS ad, para tratamento de usuários de álcool e outras drogas. 0 município ainda oferece atendimento de urgência/emergência em saúde mental no Hospital Regional, e um serviço independente de tratamento para usuários de álcool e outras drogas, denominado de Fundação Belo Amor (FBA).

O índice crescente do consumo de álcool e outras drogas no município e cidades circunvizinhas torna-se uma preocupação constante enquanto problema de saúde e segurança pública. Caicó tem uma posição geográfica privilegiada de acesso/rota de entorpecentes para a região do Seridó, sendo reconhecido atualmente $\mathrm{o}$ uso alarmante de crack em todas as classes sociais.

De acordo com a Organização Mundial de Saúde (OMS), o uso e o abuso de drogas são uma grave preocupação de saúde pública presente em mais de $10 \%$ das populações dos centros urbanos de todo o mundo, onde a confirmação do papel destrutivo dessas substâncias psicoativas aponta para a necessidade de respostas políticas coerentes e efetivas. ${ }^{2}$ Neste sentido, a implementação de CAPSad e/ou de programas voltados para o público usuário nos estados brasileiros surgiram com o intuito de promover a redução de danos, a reabilitação e reinserção social dos usuários, enfatizando a atenção comunitária articulada às redes sociais e a outros serviços de saúde. ${ }^{1}$

A modalidade de CAPSad caracteriza-se como centro especializado no atendimento de usuários de álcool e outras drogas, sendo previsto para cidades com mais de 200.000 habitantes ou cidades que, por sua localização geográfica ou cenários epidemiológicos importantes, necessitem deste serviço para dar resposta efetiva às demandas de saúde mental. ${ }^{2}$

Além da proposta deste serviço, é fundamental prover subsídios para o desenvolvimento de ações de prevenção primária ao uso de álcool e outras drogas, buscando trabalhar, juntamente com os usuários e seus familiares, os preconceitose as formas protetoras contra a influência dos fatores de risco para o uso/dependência de substâncias psicoativas.?

Desta forma, torna-se essencial capacitar equipes de saúde da família e modificar os currículos dos cursos de graduação na área da saúde, demandando abordagens reflexivas sobre a assistência oferecida aos usuários de álcool e outras drogas, dentro de uma perspectiva de intervenção precoce e de redução de danos. ${ }^{2}$

O Planto Integrado de Enfrentamento ao Cracke Outras Drogas é uma iniciativa recente do Ministério da Saúde que objetiva estruturar, integrar, articular e ampliar as ações voltadas à prevenção do uso, tratamento e reinserção social de usuários de crack e outras drogas, contemplando a participação dos familiares e a atenção aos públicos vulneráveis (crianças e adolescentes, população em situação de rua). ${ }^{3}$

Diante do exposto, buscou-se estabelecer uma aproximação dos discentes do Curso de Graduação em Enfermagem, da Universidade do Estado do Rio Grande do Norte (UERN), Campus do Seridó, com os usuários e serviços de saúde mental. Objetivou-se relatar a experiência vivenciada pelos acadêmicos de enfermagem na FBA, tendo em vista a contribuição deste percurso/movimento na construção de novos conhecimentos, somando-se aos conteúdos transversais de saúde mental ao longo do curso.

\section{APROXIMAÇÃO COM O SERVIÇO DE SAÚDE MENTAL}

Este trabalho representa uma experiência acadêmica realizada entre os meses de junho e julho de 2010, durante a oferta no sexto período da disciplina Políticas Públicas em Saúde Mental, compreendendo momentos de captação da realidade nos serviços de saúde mental da rede, e de visitas/intervenções no serviço com a participação/envolvimento dos usuários. 
A FBA está localizada no Km 103 da BR 427, município de Caicó/RN, representando um serviço filantrópico que funciona desde 2004, realizando um trabalho fundamentado na abstinência e reabilitação para usuários de álcool e outras drogas. São desenvolvidas atividades laborais cotidianas, trabalho espiritual/religioso e execução de atividades rurais (ordenha, cuidado com animais, pesca), conforme a aptidão de cada usuário. Trata-se de um sítio com capacidade para abrigar 25 usuários do sexo masculino, com idade superior a 18 anos.

Para a construção deste artigo, foi realizada uma revisão de literatura na biblioteca do Ministério da Saúde e em periódicos de enfermagem dispostos em meio eletrônico, além do diário de campo produzido durante a vivência.

\section{A MATERIALIZAÇÃO DA VIVÊNCIA}

Este relato é apresentado em três par tes referentes aos respectivos momentos de vivência no serviço (Captação da Realidade; Interação Discentes-Usuários e A Problemática do Uso/Abuso de Drogas), apresentados a seguir.

\section{Captação da Realidade}

0 primeiro momento da vivência na FBA compreendeu a captação da realidade, estratégia metodológica de aproximação com a instituição, que possibilitou aos discentes: o reconhecimento do cotidiano do serviço; as dificuldades e desafios enfrentados; o perfil sócio-demográfico dos usuários; e as necessidades prementes a serem trabalhadas na instituição, estas adaptadas/revistas a cada encontro.

A FBA apresenta uma proposta terapêutica baseada em trabalhos individuais e/ou em grupos, e terapias ocupacionais que envolvem a dinâmica de um sítio, tais como cuidados domésticos, de agricultura, pecuária e pesca. Pretende-se alcançar a reabilitação dos usuários através da abstinência e da ocupação em atividades cotidianas que exigem disciplina, compromisso, responsabilidade e reflexão, sem necessitar do uso de medicamentos ou qualquer outro tipo de tratamento realizado em âmbito hospitalar.

0 tempo mínimo de tratamento nesta instituição é de 12 meses, em que o usuário se compromete a obedecer regras do serviço através da assinatura de um termo de responsabilidade na admissão. Os quatro primeiros meses exigem permanência exclusiva no serviço, sem o direito de receber familiares e/ou amigos. Posteriormente, são liberadas as visitas nos domingos, e a visitas quinzenais ao convívio familiar no lar. As pessoas que se dispõem a ingressarem neste serviço, só podem ir de livre e espontânea vontade, assim como só lá permanecem até a hora que desejarem.

Conforme informações obtidas pela coordenação da FBA, uma média de 350 usuários de álcool e outras drogas já passaram pelo serviço, com um longo histórico de desistência e recaídas, dificuldades de convivência nos primeiros dias de "internação", a resistência em aderir às atividades terapêuticas propostas pelo serviço e o escasso apoio social e financeiro.
No período da vivência encontravam-se na instituição 17 usuários do sexo masculino, na faixa etária entre 19 e 50 anos, alguns de outros estados. 0 tempo mínimo e máximo dos usuários na FBA eram, respectivamente, 2 dias e 12 meses. A dependência química ao álcool e ao crack constituiu o principal motivo da procura destas pessoas ao tratamento, existindo casos de recaída (maioria) e de reinternação em alguns casos.

Mediante as informações obtidas no serviço, os discentes puderam planejar as intervenções a serem desenvolvidas. A realização de atividades de educação em saúde, cujo enfoque seria "as consequências do uso de drogas", logo foi descartada, uma vez que continuamente os usuários recebiam esse tipo de informações pela visita de outras instituições e colaboradores, e também por considerar que os mesmos tem muito mais a relatar que ouvir, dada a condição de dependência química.

Pensou-se em trabalhar alguma atividade que dinamizasse a rotina do serviço e de certa forma pudesse contribuir para o processo de reabilitação dos usuários. Estas atividades serão descritas a seguir.

\section{Interação Discentes-Usuários}

A estratégia pensada no segundo encontro foi, inicialmente, de conhecer os usuários por meio de uma técnica de apresentação, em que cada pessoa ficou responsável por apresentar o colega do lado com o uso de uma imagem (recorte de revista) que o representasse. Neste momento, os discentes puderam se aproximar do nível de conhecimento, intimidade e tolerância de convivência entre os usuários.

Posteriormente, foi proposta a realização de uma oficina de imagens intitulada "Tenda do Conto". Nesta atividade, os usuários selecionaram recortes de revistas (imagens) que identificavam, respectivamente, o passado, o presente e as perspectivas para o futuro.

Em cada recorte apresentado, foram comentados o seu significado e sua representação. A singularidade expressa nas imagens e na fala dos usuários configurou-se em um momento de compartilhamento de diferentes e semelhantes experiências de vida, que através da socialização verbal e/ou visual, permitiu uma troca de saberes e práticas cotidianas de sofrimento e superação, apontando uma admirável capacidade de mudança do homem.

Nesse sentido, valorizaram-se os trabalhos com grupos e as oficinas de arte-terapia que abordam conteúdos psíquicos e simbólicos, enquanto atividades terapêuticas a serem desenvolvidas nos serviços de reabilitação psicossocial dos usuários de álcool e outras drogas, principalmente quando em parcerias com instituições e grupos sociais comunitários, a exemplo das universidades ${ }^{4}$ e da experiência que se apresenta, que representam meios de expressão individual e coletiva de essencial valor social e material.

A discussão na "Tenda do Conto" referente ao passado foi associada ao relato de um mundo imerso por farras, curtição e dependência química por álcool e outras drogas, trazendo 
como consequências a perda de laços sociais e familiares e a inserção no mundo do crime. Esta última foi entendida como um fator responsável por provocar a retomada de uma posição em relação a si mesmos, quando buscaram a fuga das drogas ao entrarem na FBA, situações também vivenciadas por outros usuários. ${ }^{5}$ As imagens elegidas para esse momento incluíram prisões e assassinatos, roubos, bebidas alcoólicas, apreensão de drogas, entre outros.

Resultados encontrados em uma pesquisa ${ }^{6}$ apontam que a informação insatisfatória ou ineficiente sobre o uso de drogas enfocando apenas os aspectos desejados das drogas, como "curtição" e "barato", desperta na população a curiosidade que associada à informação vaga sobre os prejuízos da dependência, influenciam o consumo. Entretanto, a repercussão social causada pelos impactos negativos da desestruturação na vida social e pessoal do usuário, como o envolvimento no crime e na prostituição, acaba por suscitar representações repelitivas ao uso de drogas pela população. ${ }^{6}$ Logo, estas representações merecem ser destaque na mídia e em instituições de educação e de saúde, como forma de sensibilizar a comunidade.

O tempo presente esteve relacionado ao momento de reabilitação na FBA. Os usuários referiram-se a este como um estágio de renovação da mente, de aprendizado, de dedicação às "tarefas terapêuticas", de reflexão, fé, apoio e reconciliação com os familiares, momento percebido como difícil e desafiador, porém necessário ao processo de reestruturação de suas vidas. As imagens referentes a este estágio incluíram os discursos de superação, imagens religiosas, paisagens naturais.

Percebeu-se que a FBA tem como um dos seus objetivos colocar o usuário na posição de protagonista do seu tratamento. Desta forma, os esforços para manter-se no tratamento dependem das relações entre os colegas (companheirismo, apoio), familiares e a própria vontade de se recuperar. Neste ínterim, as atividades focalizadas em intervenções sociais, atribuindo funções, direitos e responsabilidades aos indivíduos em tratamento, fazem com que novos valores sejam criados/ resgatados pelos usuários, como a espiritualidade, responsabilidade, solidariedade, honestidade e amor $^{7}$, valores que os motivam à reabilitação.

Em relação ao futuro, a reinserção no mercado de trabalho e a reconstrução da família mediante a abstinência das drogas são algumas metas traçadas pelos usuários. Reparar os erros cometidos no passado, almejando uma vida tranquila, com confor to e bem-estar, é encarado como alicerce para o alcance desses objetivos. Todavia, podem sofrer certo grau de dificuldade se acompanhada da falta de apoio social e familiar, gerando uma relação de medo ou frustação diante de situações limítrofes à conquista do que foi almejado ${ }^{8}$, e até mesmo suscitar recaídas.

Nesse sentido, vale ressaltar que a abstinência é apenas o primeiro passo na recuperação. Ao retomar suas vidas, os usuários são constantemente expostos a situações que os remetem ao consumo de álcool e outras drogas. Por isso, manterse em abstinência no futuro exige uma profunda revisão de condições ambientais, reestruturação de atividades e revitalização de interesses, a fim de reconhecer e conscientizarse dos desafios que a reabilitação implica. ${ }^{5}$

Ao término da "Tenda do Conto", as imagens escolhidas para representar o futuro dos usuários foram fixadas em um painel e expostas no espaço do serviço, com o intuito de colaborar com a proposta da FBA por meio do estímulo ao processo de reabilitação mediante o desejo de alcançar as metas fixadas.

\section{A Problemática do Uso/Abuso de Drogas}

Propostas para realização de outras atividades foram acolhidas pelos discentes, e, no terceiro e último dia da vivência, foi realizada a "Dinâmica dos Problemas", momento em que os usuários puderam desabafar sobre alguns sentimentos ou situações vivenciadas que os induziram ao consumo de álcool e outras drogas, seja na iniciação ou em recaídas.

Esta técnica foi pensada a partir da necessidade exposta pelos usuários de desenvolverem alguma atividade que minimizasse a ansiedade. Neste sentido, foi idealizado que este exercício de desabafo fosse capaz de promover um maior alívio nas tensões provindas do cotidiano da reabilitação, sendo ainda distribuídos cadernos (diários) e lápis para cada usuário dar continuidade a este movimento de forma individual ou coletiva no dia-a-dia. Além disso, constitui-se em uma forma de visualizarem continuamente a sua recuperação/reabilitação.

Reforça-se a importância do suporte do serviço em questão e a valorização das atividades terapêuticas. Como encontrado em outra pesquisa ${ }^{8}$, o desabafo pareceu transcender uma relação de vínculo com quem escuta, para criar um ambiente terapêutico, onde o relato de uma penosa trajetória, permeada por arrependimentos, constituiu-se em um momento de reflexão e reconstrução de conceitos.

Assim sendo, a dinâmica ocorreu da seguinte forma: foram disponibilizados dez balões contendo problemas relativos a situações e/ou sentimentos envolvidos com o consumo de álcool e outras drogas. Em seguida, solicitou-se que cada usuário fizesse a leitura do tema proposto no interior do seu balão e discorresse sobre a implicação daquela situação ou sentimento em sua vida. Os temas incluíram: ansiedade; antigas amizades; conflitos familiares; drogas e bebidas alcoólicas; desesperança; medo; pressão social; preconceito da sociedade, familiares ou consigo mesmo; e solidão.

Trocas de experiências e situações já vivenciadas pelos usuários foram levantadas, bem como especulações sobre o que irão encontrar ao retornarem às suas famílias e à vida cotidiana fora da FBA. Sugestões, alternativas e atitudes de superação a serem tomadas diante das problemáticas dinamizadas se tornaram peças-chave nos discursos dos usuários, como forma de abrandar a expressão de angústia e apoiar os colegas em processo de reabilitação.

Neste momento, percebeu-se uma forte manifestação de religiosidade e espiritualidade pelos usuários, cuja relação foi sugerida por pesquisas como uma prática cultural de remissão dos pecados, 
de culpa e de proteção. Além disso, infere-se que os dependentes químicos em processo de reabilitação encontram no apoio dos familiares e amigos o alicerce para reestruturação de suas vidas. ${ }^{5}$

Preventivamente, alerta-se que o consumo de drogas licitas, a exemplo do álcool, seja o principal artifício que encoraja o uso de drogas ilícitas, sendo considerada a "porta de entrada" para a iniciação nas drogas. ${ }^{9} 0$ alcoolismo parece, então, ganhar destaque enquanto fator/condição de risco, sendo de fundamental importância a atuação da Enfermagem e da ESF na realização de atividades de educação em saúde na comunidade com vistas à prevenção, isto é, ações que pretendam minimizar os fatores de risco sobre a vulnerabilidade dos indivíduos. ${ }^{10}$

Nesta perspectiva, além do etilismo, reconhece-se que a desestruturação familiar e/ou rejeição, falta de apoio, angústia, raiva e ansiedade são propulsores para o uso e abuso de drogas ilicitas na sociedade, bem como a influência das antigas amizades, os ápices de tristeza e/ou felicidade e o preconceito consigo mesmo, induzindo recaídas. $5,8,9$

Foi encontrado em um estudo ${ }^{11}$ que, entre os fatores que comprometem a autoeficácia dos usuários em processo de reabilitação, diante da resistência ao álcool e outras drogas (recaídas), estão: as emoções negativas; as situações relacionadas com o prazer; as perspectivas retraídas em relação ao tratamento; e, principalmente, deparar-se com situações nas quais, habitualmente, consumiam drogas.

Logo, entende-se que a prevenção de recaídas deve ser trabalhada com base nessas situações e conceitos. A experiência que se apresenta e a literatura disponível já apontam alguns subsídios e fatores de riscos nos quais os usuários se sentem vulneráveis e, portanto, merecem ser consideradas pelos profissionais de saúde e a família no que se refere à sensibilização.

A aproximação com a realidade vivenciada pelos usuários fomentou no imaginário dos acadêmicos um movimento de retroalimentação de ideias, com vistas à construção de estratégias/ intervenções na comunidade e nos serviços de saúde relacionados ao uso/abuso de álcool e outras drogas. Destaca-se a relevância de ações fundamentadas na perspectiva da integralidade, a fim de garantir não apenas o atendimento ao usuário e sua família, mas também de possibilitar caminhos para a reabilitação psicossocial.12

Sobressaiu-se, então, a inquietação com relação à prevenção por meio do rastreamento de situações de risco (identificadas por dinâmicas) e de atividades de educação em saúde com vistas à promoção em saúde, enfocando as relações interpessoais.

De acordo com as Diretrizes Curriculares Nacionais (DCN), os currículos dos cursos de graduação na área da saúde devem assumir espaços de participação, reflexão e articulação entre educação superior e serviços de saúde, objetivando a formação dos egressos-profissionais com base na promoção, prevenção, recuperação e reabilitação. ${ }^{13}$

Valoriza-se, neste sentido, que a formação do enfermeiro abarque disciplinas de saúde mental na sua grade curricular, como forma de instrumentalizar e profissionalizar o acadêmico nesta área de atuação. Faz-se necessário redirecionar conteúdos e metodologias de ensino ainda presente em algumas escolas de enfermagem que priorizam a doença e a conduta terapêutica baseada na psiquiatria tradicional, ${ }^{14}$ como forma de responder à nova tendência das práticas em saúde mental imbricada pelos pressupostos da Reforma Psiquiátrica.

No caso espećfico da proposta de formação do enfermeiro egresso do Curso de Graduação em Enfermagem, Campus Caicó (UERN), o Projeto Pedagógico do Curso (PPC) estabelece que o conteúdo de saúde mental seja oferecido de forma transversal nas várias disciplinas espećíficas, não existindo uma só disciplina com essa finalidade. A experiência que se apresenta, ainda de forma inicial, aponta lacunas nos conteúdos de enfermagem em saúde mental/psiquiatria e, consequentemente, na construção de conhecimento nesta área. ${ }^{15}$

Diante desta demanda, houve a oferta da disciplina optativa "Políticas Públicas de Saúde Mental" no semestre 2010.1. Esta disciplina tem como objetivo principal ressignificar a loucura enquanto fenômeno social e compreender o processo de Reforma Psiquiátrica vivenciada no Brasil. Esta iniciativa mostrou-se favorável para a construção de competências dos graduandos naárea da saúde mental, especialmente por trabalhar a politica nacional de saúde mental, e ainda oportunizar a inserção nos serviços de saúde mental do município. Iniciativas como esta permitem que a relação entre discentes e usuários seja construída a partir da abordagem das necessidades e subjetividades de cada sujeito, cujas estratégias de enfretamento são elaboradas com base no diálogo.

Como ressaltado pelo Ministério da Saúde, todo esse processo de reabilitação dos usuários de álcool e outras drogas envolve planejamento, implantação e implementação de múltiplas estratégias voltadas para a redução dos fatores de vulnerabilidade e risco espećificos, e o fortalecimento dos fatores de proteção. Isso implica, necessariamente, a inserção comunitária das práticas propostas, com a colaboração de todos os segmentos sociais disponíveis. Para tanto, a lógica da redução de danos deve ser considerada como estratégica ao planejamento de propostas e ações preventivas. ${ }^{2}$

A vivência concretizada na FBA despertou a ideia de resgate e promoção da educação em saúde, cuja pertinência potencializa a redução de custos junto aos vários contextos da assistência, favorece a compreensão abrangente dos problemas relativos ao uso/abuso de drogas, além de desenvolver autonomia e corresponsabilidade dos usuários diante do seu processo saúdedoença.

\section{CONSIDERAÇÕES FINAIS}

Esta experiência contribuiu para que os acadêmicos percebessem a relevância do fenômeno do uso/abuso de drogas sob a ótica dos usuários (problemática de vida) e lançassem novos olhares sobre as estratégias de prevenção e promoção à saúde, a serem desenvolvidas na comunidade e nos serviços de saúde do município. 


\section{Formação do acadêmico de enfermagem}

Brito AAC, Silva DS, Azevedo DM

Nesta perspectiva, a formação em saúde destaca a importância de atividades que contemplem a relação "teoriaprática-teoria", objetivando um ambiente de aprendizagem capaz de suscitar nos alunos o despertar para a solução de problemas, o contato com situações específicas e o trabalho em grupo. Considera-se, ainda, a visualização de um processo de trabalho em saúde extremamente rico e plural, dada a lógica do cuidado na perspectiva da saúde mental.

Os discentes identificam, ainda, que esta metodologia de ensino colaborou para a construção de conceitos e experiências importantes no auxílio da formação profissional generalista, crítica e reflexiva do enfermeiro egresso da UERN, Campus Caicó.

\section{REFERÊNCIAS}

1.Ministério da Saúde (BR). Reforma psiquiátrica e política de saúde mental no Brasil. Brasília(DF); 2005.

2.Ministério da Saúde (BR). A política do Ministério da Saúde para atenção integral a usuários de álcool e outras drogas. Brasília (DF); 2003.

3.Decreto n.7.179, de 20 de maio de 2010. Institui o Plano Integrado de Enfrentamento ao Crack e Outras Drogas, cria o seu Comitê Gestor, e dá outras providências. Diário Oficial da República Federativa do Brasil, Brasília (DF), 21 maio 2010: Seção 1: 43.

4.Pinho PH, Oliveira MAF, Vargas D, Almeida MM, Machado AL, Silva ALA, et al. Reabilitação psicossocial dos usuários de álcool e outras drogas: a concepção de profissionais de saúde. Rev Esc Enferm USP. 2009; 43(2): 1261-66.

5.Rigotto SD, Gomes WB. Contextos de abstinência e de recaída na recuperação da dependência química. Psicol. 2002 jan/abr; 18(1): 95106.

6.Sanchez ZVDM, Oliveira LG, Nappo AS. Razões para o não-uso de drogas ilícitas entre jovens em situação de risco. Rev Saude Publica. 2005; 39(4): 599-605.

7.Sabino NDM, Cazenave SOS. Comunidades terapêuticas como forma de tratamento para a dependência de substâncias psicoativas. Estud Psicol. 2005 abr/jun; 22(2):167-74.

8.Azevedo DM, Miranda FAN. Práticas profissionais e tratamento ofertado nos CAPSad do município de Natal-RN: com a palavra a família. Esc Anna Nery. 2010 jan/mar; 14(1): 56-63.

9.Crives MNS, Dimenstein M. Sentidos produzidos acerca do consumo de substâncias psicoativas por usuários de um programa público. Saude Soc. 2003 jul/dez; 12(2): 26-37.
10. Silva SED, Vasconcelos EV, Padilha MICS, Martini JG, Backes VMS. A educação em saúde como uma estratégia para enfermagem na prevenção do alcoolismo.Esc Anna Nery. 2007; 11(4): 699-705.

11.Kantorski LP, Lisboa LM, Souza J. Grupo de prevenção de recaídas de álcool e outras drogas. Rev Eletr Saude Mental Álcool Drogas. [periódico on-line]. 2005; [citado 2010 jul 15]; 1(1): 1-15. Disponível em: http:// pepsic.bvsalud.org/scielo.

12.Miranda FAN, Simpson CA, Azevedo DM, Costa SS. 0 impacto negativo dos transtornos do uso e abuso do álcool na convivência familiar. Rev Eletr Enferm. [on-line]. 2006; [citado 2010 jul 22]; 8(2): 222-32. Disponível em: http://www.fen.ufg.br/revista/revista8_2/v8n2a07.htm

13.Barbosa ECV, Viana LO. Um olhar sobre a formação do enfermeiro/ docente no Brasil. Rev Enferm UERJ. 2008 jul/set; 16(3): 339-44.

14.Silva ATM, Souza JS; Silva CC; Nóbrega MML; Oliveira Filha M; Barros S, et al.Formação de enfermeiros na perspectiva da reforma psiquiátrica. Rev Bras Enferm. 2004 nov/dez; 57(6): 675-78.

15.Silva DS, Azevedo DM. A reforma psiquiátrica na visão de quem cuida: percepções de profissionais do serviço residencial terapêutico. Esc Anna Nery. No prelo 2012. 